\title{
Relativistic Heisenberg principle for vortices of light from Planck to Hubble scales
}

\author{
F. Tamburini $\odot,{ }^{1, *}$ Ignazio Licata $\odot,{ }^{2,3,4, \dagger}$ and B. Thidé $\odot^{5, \$}$ \\ ${ }^{1}$ ZKM-Zentrum für Kunst und Medientechnologie, Lorentzstrasse 19, D-76135, Karlsruhe, Germany \\ ${ }^{2}$ Institute for Scientific Methodology (ISEM), Palermo, I-90146, Italy \\ ${ }^{3}$ School of Advanced International Studies on Theoretical and Nonlinear Methodologies of Physics, Bari, I-70124, Italy \\ ${ }^{4}$ International Institute for Applicable Mathematics and Information Sciences (IIAMIS), B.M. Birla Science Centre, Adarsh Nagar, \\ Hyderabad 500 463, India \\ ${ }^{5}$ Swedish Institute of Space Physics, Ångström Laboratory, P. O. Box 537, SE-751 21, Sweden
}

(Received 1 June 2020; accepted 24 July 2020; published 1 September 2020)

\begin{abstract}
We discuss, in the relativistic limits, Heisenberg's uncertainty principle for the electromagnetic orbital angular momentum (OAM) of monochromatic fields. The Landau and Peierls relativistic approach applied to the Heisenberg indetermination between the azimuthal angle $\Delta \phi$ and the OAM state $\Delta \ell$, when a limit speed is present, exposes the conceptual limits of a possible formulation of the uncertainty principle for OAM states as it leads to the Ehrenfest's paradox of the rotation of a rigid disk in special relativity (SR). To face this problem, in a way similar to Einstein's discussion of a rotating rigid disk, one has to adopt a general relativistic approach and include the limits from smallest and largest scales present in nature, such as the Planck scale and the Hubble horizon.
\end{abstract}

DOI: 10.1103/PhysRevResearch.2.033343

\section{INTRODUCTION}

The classical electromagnetic (EM) field transports energy $E$ and momentum $\mathbf{P}$ as well as angular momentum $\mathbf{J}$. This is the case also at the quantum level, where it finds a precise and comprehensive description through the second-quantization formalism of quantum electrodynamics (QED) [1] or in the first quantization language via the photon wave function by using the Majorana-Wigner approach to quantum electrodynamics, based on the Riemann-Silberstein formalism in a direct correspondence to QED [2].

The total angular momentum $\mathbf{J}=\mathbf{S}+\mathbf{L}$ is transported in two different forms: The first, $\mathbf{S}$, is the spin angular momentum (SAM) related to the spin of the photon. In a fully covariant approach, the photon wave function has a total spinorial representation of rank 6 , equivalent to a spinor of rank 2 for each coordinate, i.e., a vector giving photons an intrinsic spin $s=1$ with associated helicity quantum numbers, $\lambda= \pm 1$. The second quantity, $\mathbf{L}$, is the orbital angular momentum (OAM), associated with the phase profile of the light beam, and therefore directly depends on the spatial coordinates [3-6]. Since a photon cannot be associated with a rest reference frame, the splitting $\mathbf{J}$ into the two gauge-invariant

\footnotetext{
*fabrizio.tamburini@gmail.com

†ignazio.licata3@gmail.com

¥bt@irfu.se
}

quantal observables $\mathbf{S}$ and $\mathbf{L}$ is not possible in general [7]. The distinguishability of the spin and the orbital angular momentum requires that the "spin" and "coordinate" properties of the wave functions should be independent of each other, but the problems of the photon localizability make it impossible to construct a representation in the momentum and coordinate at the same time and in an immediate way. Moreover, the photon wave function must obey the transversality condition, since the photon is a zero-rest-mass particle [8]. Because of the transversality condition, this function cannot simultaneously specify all the values of each of its vectorial components and therefore the orbital angular momentum and the spin cannot be separated. For instance, $\mathbf{J}$ might have a different representation in terms of $\mathbf{S}$ and $\mathbf{L}$ when the light beam propagates in an inhomogeneous medium. However, in the case of light beams propagating in vacuum, it is possible to separate the two commuting operators $S_{z}$ and $L_{z}$ of the total angular momentum $z$ component, obtained by projecting the two quantities $\mathbf{S}$ and $\mathbf{L}$ onto the propagation axis of the beam $z$. Quantum field theory and QED confirm this picture, in which each individual photon carries an amount of SAM, quantized as $S=\sigma \hbar$, where $\sigma= \pm 1$ and can additionally carry OAM, quantized as $L=\ell \hbar, \ell=0, \pm 1, \pm 2, \ldots, \pm N$ [7,9]. This property of EM waves and photons has been confirmed experimentally [10-17] and discussed theoretically $[2,18,19]$. The uncertainty principle for OAM relates the phase and photon number or the angular position and orbital angular momentum was discussed in Refs. [20-22].

\section{OAM-HEISENBERG AND THE EHRENFEST PARADOX}

Let us consider cylindrical OAM beams, for the sake of simplicity and without loss of generality [23]. A particular 
class of cylindrical beams carrying OAM is, for example, Laguerre-Gaussian (L-G) beams, which are characterized by helical wave fronts carrying a well-defined value $\ell \hbar$ of OAM per photon for any EM frequency and $\ell \in \mathbb{Z}$, viz., an integer $[24,25]$. As these beams have cylindrical symmetry, all physical properties are periodic functions of an angular position. For any given integer OAM value $\ell \in \mathbb{Z}$, optical vortices (OVs) are found along the symmetry and propagation axis $z$ of the beam. On this axis, the field amplitude goes to zero and the phase is undetermined. The simplest formulation of a cylindrical vortex beam propagating along the $z$ axis of a cylindrical coordinate set $(r, \phi, z, t)$ depends on the angle $\phi$, OAM $\ell$, and amplitude $u(r, z, t)$,

$$
\Psi(r, \phi, z, t)=u(r, z, t) e^{i \ell \phi} .
$$

The angular observables and the eigenvalues of the angle operator $\hat{\phi}_{\phi}$ are found in the range $[\phi, \phi+2 \pi)$ and the angle operator is defined as $\hat{\phi}_{\phi}=(\phi) \bmod 2 \pi$.

However, the orbital angular momentum operator, $\hat{\mathbf{L}}=\hat{\mathbf{r}} \times$ $\hat{\mathbf{p}}$, projected onto the propagation axis $z$ of the beam,

$$
\hat{L}_{z}=-i \hbar\left(x \frac{\partial}{\partial y}-y \frac{\partial}{\partial x}\right)=-i \hbar \frac{\partial}{\partial \phi},
$$

and assumes discrete values, $L_{z}=\ell \hbar$.

The Heisenberg uncertainty principle can be formulated as follows: Any Hermitian operator, $\hat{A}$, representing a physical observable, has expectation value $A=\left\langle\psi^{*}(q)|\hat{A}| \psi(q)\right\rangle$. Strictly speaking, the probability density is $|\psi(q)|^{2}$. The wave function $\psi(q)$ can in most cases be an $L^{2}$-integrable function and the total probability can be normalized to unity. In some cases, the integral may diverge and the probability cannot be normalized in the whole parameter space, but the ratio of the values of the probability at two different points of configuration space can be, so determining the relative probability distribution. Because of this, the uncertainty in the measurement of the quantity $A$ is represented by the interval $\Delta A$, i.e., $(\Delta A)^{2}=\left\langle\psi^{*}(q)\left|(\hat{A}-A)^{2}\right| \psi(q)\right\rangle$. If we consider another operator $\hat{B}$ associated with the observable $B$, the uncertainty principle for the two variables $A$ and $B$ in a general commutator formulation is $[\hat{A}, \hat{B}]=i \hat{C} \hbar$, namely,

$$
\Delta A \Delta B \geqslant \frac{\hbar}{2}\left|C_{0}\right|,
$$

where $C_{0}$ is the mean value of the general commutator $\hat{C}$. For conjugate variables, the mean value of the general commutator is $C_{0}= \pm 1$. As there is no direct formulation of a Hermitian operator for the phase of the photon, we will use the geometrical properties of the beam to discuss Heisenberg indetermination relationships instead of building a Hermitian operator related to the phase through the two operators " $\widehat{\sin }$ " and " $\widehat{\cos }$ " that are trigonometric functions of the phase and do not have an immediate physical interpretation [26,27]. Thus, for OAM states, the commutator $\hat{C}$, used in the generic formulation of Heisenberg's principle, must be a periodic function of the azimuthal angle $\phi$. This implies that $C_{0}=1-2 \pi P(\phi)$, yielding [22]

$$
\Delta \phi_{\phi} \Delta L_{z} \geqslant \frac{1}{2} \hbar|1-2 \pi P(\phi)|
$$

The azimuthal momentum quantum operator is described in terms of a density of the orbital angular momentum $\hat{l}_{z}$ and the length of the radius vector $r$,

$$
\hat{p}_{\phi}=-i \frac{\hbar}{r} \frac{\partial}{\partial \phi}=\frac{\hat{l}_{z}}{r} .
$$

Anyway, Heisenberg's uncertainty relationship has been formulated in the language of classical, nonrelativistic, quantum mechanics. Because the photon is an ultrarelativistic particle $[7,28]$, one has to pay attention also to the existence of a limit speed, which is the speed of light $c$. In 1930, Landau and Peierls discussed Heisenberg's relationship when a limit speed is included,

$$
\left(v^{\prime}-v\right) \Delta p \Delta t \geqslant \frac{\hbar}{2},
$$

and because of the existence of the finite limit speed $c$ the absolute value of the difference $\left(v^{\prime}-v\right)$ cannot be larger than $c$. In the ultrarelativistic limit, when $\left(v^{\prime}-v\right) \sim c$, one obtains a relationship involving momentum and time. The coordinate indetermination is then translated into an indetermination of the measurement in time, $\Delta p \Delta t \geqslant \hbar / 2 c$. In a more general formulation,

$$
\Delta A \Delta t\left(\frac{\Delta B}{\Delta t}\right) \geqslant \frac{\hbar}{2}\left|C_{0}\right|
$$

from Eq. (4), and assuming $\Delta A=\Delta L_{z}$, one obtains, for a given length of the radius $r$,

$$
\frac{1}{r} \Delta L_{z} \Delta t \geqslant \frac{1}{2} \hbar\left(\frac{1}{\Omega_{\phi}}\right)|1-2 \pi P(\phi)| \sim \frac{\hbar}{2 c}
$$

and $|1-2 \pi P(\phi)| \sim r \Omega_{\phi} / c$, which implies that in a local relativistic limit the uncertainty is not well defined, since $P(\phi) \in[0,2 \pi)$. In the frequency domain, instead,

$$
\Delta L_{z} \Delta t \geqslant \frac{\hbar}{2}\left(\frac{r}{\frac{d \phi}{d t}}\right)|1-2 \pi P(\phi)| \sim \frac{\hbar}{2} \frac{r}{c}
$$

and $|1-2 \pi P(\phi)| \sim d \phi / c d t=\Omega_{\phi} / c$ and in the ultrarelativistic limit $r \sim c / \Omega_{\phi}$. For integer OAM modes, one sets $\Delta L_{z}=\hbar \Delta \ell$. Heisenberg's uncertainty relationship for OAM modes then becomes

$$
\Delta \ell \Delta t \geqslant \frac{1}{2}\left(\frac{r}{\Omega_{\phi}}\right)|1-2 \pi P(\phi)| \sim \frac{1}{2} \frac{r}{c}
$$

and the indetermination of the OAM state in the time interval $\Delta t$ must be larger than half the distance from the phase singularity position in which is measured the vortex pattern, $r$, divided by the speed of light $c$, calculated within a phase variation of $2 \pi$. The upper limit in the ultrarelativistic case is given by the rightmost member of Eq. (10). Notice that on the right-hand side of Eq. (10) $r$ is defined up to the positive infinity and Heisenberg's relationship leads to the Ehrenfest paradox of the rotation of a rigid disk in SR [29-31]. In this paradox, Einstein showed that if one measures a set of rods aligned along the periphery of the disk, it should appear contracted. The observer is comoving with the disk rotation; the rods, because of the relativistic contraction, should appear contracted; and naively a larger number of them would fit around the disk's border, indicating that the 
disk border would have a circumference larger than $2 \pi r$. The geometry is non-Euclidean, with an expected Lorentz contraction of $\sqrt{1-\Omega_{\phi}^{2} r^{2} / c^{2}}$ and rotation velocity $v_{\phi}=\Omega_{\phi} r$. If $c$ is the limit speed, then $v_{\phi} \leqslant c$ implies $\Omega_{\phi} r \leqslant c$ and for $r \rightarrow \infty$ the angular velocity must decrease or the clocks in the periphery of the rotating body go slower, in agreement with SR. The paradox is solved by using general relativity (GR), where - as in special relativity — no rigid bodies exist and in GR spacetime is described by curved Riemannian geometries when gravitating bodies or accelerations are present. This recalls the geometry of the EM field of, e.g., a L-G mode with OAM $\ell$ (and radial number $p=0$ ) where the phase of the field does not depend explicitly on the distance of the optical singularity. The effect of vorticity can be ideally measured at large distances, up to infinity in the plane orthogonal to that of the beam propagation, as the intensity pattern of L-G beams have always a finite value tending to zero at infinity, decaying exponentially. To avoid superluminal velocities, one first has to use the angular momentum operator in a relativistic approach avoiding to interpret the local twisting of the field in terms of angles associated to mechanical rigid rotations: OAM states of light are related to the local angular momentum of the field and not to a rigid rotation around the $z$ axis. One has to use GR for a coherent description of the twisted EM field.

To prove this, let us proceed with a reductio ad absurdum. By applying in a Minkowski spacetime a general coordinate transformation to make $L_{z}$ locally disappear, we must calculate this relationship in a rigid corotating frame, rotating with angular velocity $\Omega_{\phi}=d \phi / d t$ around the $z$ axis. In the cylindrical coordinate set $(r, \phi, z, t)$, the line element $d s$ of the Riemannian geometry for this rotating flat spacetime is [28,32]

$$
d s^{2}=\left(c^{2}-\Omega_{\phi}^{2} r^{2}\right) d t^{2}-2 \Omega_{\phi} r^{2} d \phi d t-d z^{2}-r^{2} d \phi^{2}-d r^{2} .
$$

Even if this metric is locally diagonalizable, because of the equivalence principle, it cannot describe the behavior of a real gravitational field at large distances. Otherwise, one would have to break the limit of the speed of light $c$ for certain values of the radius $r$, violating causality. The mandatory condition on the metric tensor, $g_{00}>0$, implies that this particular quadratic form is valid only for distances that are in the interval $0<r<c / \Omega_{\phi}$. With this limit imposed by $c$, one can neglect the rotation of the momentary field rotation with a local gravitational field only when $r<c / \Omega_{\phi}$, limiting the spatial extension of the OAM state. By adding the causality condition in a corotating frame, one gets also an upper limit in the indetermination relationship,

$$
\frac{\hbar}{2 \Omega_{\phi}}|1-2 \pi P(\phi)| \leqslant \frac{1}{r} \Delta L_{z} \Delta t \leqslant \frac{\hbar}{2 \Omega_{\phi}},
$$

which implies $\Delta \ell c \Delta t=\Delta \ell \Delta r \sim r^{2} / 2$ and the following inequalities

$$
\frac{r}{2 \Omega_{\phi}}|1-2 \pi P(\phi)| \leqslant \Delta \ell \Delta t \leqslant \frac{r}{2 \Omega_{\phi}} .
$$

When Heisenberg's relationship is described in terms of an angle-momentum uncertainty relationship

$$
\Delta p \Delta(r \phi)=r \Delta p \Delta \phi \geqslant \frac{\hbar}{2}\left|C_{0}\right|,
$$

equivalent to that involving the projection onto the $z$ axis of the OAM operator with the $2 \pi$ periodicity, being $r \Delta p=\Delta L_{z}$,

$$
\Delta \ell \Delta E \sim \frac{c}{r},
$$

then one obtains an interesting result: In a local reference frame centered on the coordinate origin, the indetermination relationship is defined everywhere but for $r=0$, where $E=$ 0 , reflecting and preserving the topology of the EM vortex and related geometric properties [33].

Given an optical vortex of order $\ell$, a test particle is expected to rotate around the $z$ axis with angular velocity imparted by photons with frequency $v=\omega / 2 \pi$, namely $\Omega_{\phi}=\ell \hbar \omega$ following the prescription of the relativistic formulation of the orbital angular momentum. In the ultrarelativistic limit $(v \sim c)$, this would imply the existence of a limit in the indetermination of any OAM state that depends either on the time interval within the local measurement is taken or in the measure of the photon wavelength from SR, $\Delta \ell \Delta t \sim 1 / c$ and for a photon with wavelength $\lambda$ one has $\Delta \ell \Delta \lambda \sim 1$. This finds agreement with the Ehrenfest's paradox: Either the angular velocity decreases or the clocks go slower as $r$ grows or the OAM is not preserved.

In GR, the rotation around a preferred axis and corotating frames describing the gravitational field alone or coupled with the EM field and/or matter are associated with frame dragging. Frame dragging on a spinning test particle occurs also with OAM radiation in the linear regime of Einstein's equations. On a test particle, the effect induced by an OAM beam gives rise to Lense-Thirring precession of a spinning neutral particle placed along the beam axis, the precession frequency results quantized depending from the orbital angular momentum $\ell$ of the OV and sign [34,35]. Consider the BergmannThomson gravitational angular pseudotensor related to the Einstein canonical energy-momentum tensor of matter and fields, $T_{i}^{k}$, and of the pseudotensor of gravitation, $t_{i}^{k}$,

$$
K_{k}^{i}=\sqrt{|g|}\left(T_{i}^{k}+t_{i}^{k}\right),
$$

this can be decomposed as the sum of the spinorial part

$$
S^{i k l}=U^{i[k l]}-U^{k[i l]}
$$

plus the orbital one

$$
O^{i k l}=\sqrt{|g|}\left(y^{i} t^{k l}-y^{k} t^{i l}\right)
$$

of the gravitational angular momentum densities. The symbol $g$ is the metric tensor norm, $y^{i}$ is the normal coordinates, $G$ is the Newton's constant, and

$$
U^{i[k l]}=\frac{c^{2}}{16 \pi G} g^{i m} \frac{g_{m a}}{\sqrt{|g|}}\left[-g\left(g^{k a} g^{l b}-g^{l a} g^{k b}\right)\right],{ }_{b} .
$$

This means that the averaged angular momentum tensors for energy, fields averaged on a four-sphere of radius $r$, as discussed in Ref. [36] follow a behavior as $\Delta \ell \Delta r \sim r^{2} / 2$, from the formulation of Heisenberg's uncertainty principle 
and the orbital part $O^{i k l}$ is a pseudotensor. From this, we see that the orbital part does not contribute to the averaged tensors, while it is the spinorial part only that gives nonzero contribution to them. In any case, the tensors depend on a fundamental length, which is the radius $r$ and naively should be infinitesimally small to preserve the local Lorentz invariance in the indetermination principle expressed in Eq. (15). To get rid of this ambiguity derived from Ehrenfest's paradox, one should use an approach similar to that adopted by Einstein to solve this paradox, assuming that the metric is non-Euclidean. More precisely, as reported in Ref. [37], p. 032109, "Although there is no relative motion among different points on a rotating disk, each point belongs to a different non-inertial frame," implying that one should then refer to the actual quantum mechanical properties of OAM photons, measured locally, having $p=h v / c$ in an optical vortex with topological charge $\ell$ where all photons are supposed to have a well-defined value of orbital angular momentum $J_{z}=\ell \hbar$. In Fermi coordinates, these quantities hold for any observer, the value of $c$ isotropic, when measured by propagating a light beam in a small neighborhood of the observer, and the assumed constant OAM for each photon in the beam is mathematically defined in a GR framework. In gravitational fields generated by a rotating mass such as the Kerr [17,38-40], or the Gödel solutions (where the circular orbit of the maximum radius is the null geodesic) [41], one instead finds that photon OAM interacts with that of the rotating body: Both solutions belong to the class of Petrov type-D geometries, where the mixing of space and time coordinates actively imprint OAM or even to subtract OAM from light or modify the properties of the beam with an additional rotation $[17,40,42]$. Only in these cases one can apply a global transformation to counterbalance an OAM state and the pseudotensor $K_{k}^{i}$ does not vanish, reflecting the global rotation of the spacetime. Moreover, Gödel spacetimes pose additional limitations to Heisenberg principle because of the presence of closed timelike curves (CTCs). In fact, Gödel's universe is based on the sub-Riemannian geometry of the Heisenberg group and cannot hold at large scale the structure of spacetime of Einstein's general theory of relativity unless including CTCs, while it is locally permitted at the small scales of quantum theory, according to the fundamental Stone-Von Neumann theorem on the unitary and irreducible representation of the Heisenberg group in the Hilbert space including the chronology protection conjecture $[43,44]$. We conclude that the indetermination relationship depends on the geometry of spacetime and the choice of the reference frame of the observer because, being a quantum phenomenon, angles used in the first formulation of Heisenberg principle have a purely formal value and must be referred to the actual physical properties of the OAM photons.

We finally discuss some additional considerations about the indetermination of OAM states imposed by cosmological constraints. Consider an OAM beam and its momentary field distribution of the linear momentum in the static case $(\Omega=0)$. If one measures in time beams with equal phase slope and same intensity, it is not possible to determine the OAM value from it. In fact, the momentary field rotation frequency of the momentary field distribution goes as $\Omega_{m}=$ $\omega_{\text {light }} / \ell$ : The higher the OAM value, the slower the beam rotates [45], and each $[0,2 \pi]$ change of phase has the same time of switching as a whole $\ell=1$ mode, avoiding apparent superluminal motion and ideally limiting the maximum value of the OAM to that made during the whole age of the Universe. The maximum indetermination value in the estimation of the wavelength of an electromagnetic wave, $\left.\Delta \lambda\right|_{\max }$, must be smaller that the Hubble radius, the radius of the Universe horizon, $R=c / H$, related to the Hubble expansion parameter $H$, and $c$ is the speed of light. $R$ defines the size of the nearby universe that has been in causal contact with an observer. More precisely, one can assume that a photon cannot have a wavelength larger than the size of the last scattering surface, when the Universe became transparent to the radiation, $\left.\Delta \lambda\right|_{\max } \sim 1.796 \times 10^{28} \mathrm{~cm}$. In cgs units, one obtains a minimum value of the indetermination of an OAM state, and a maximum by selecting the Planck scale, $\left.\Delta \lambda\right|_{\min }=$ $1.616252(81) \times 10^{-33} \mathrm{~cm}$, which implies a maximum indetermination in the OAM value on the order of $\Delta \ell \sim 6.1872 \times$ $10^{32}$. Hence, the finiteness of our universe and the existence of a limit scale such as the Planck scale imply the existence of a minimum and maximum value on the indetermination of OAM, namely, $5.5679 \times 10^{-29}<\Delta \ell<6.1872 \times 10^{32}$. The indetermination in an OAM state of light will be zero only if the Hubble horizon is infinite, which means an infinite time after the big bang. Infinite superhorizon modes in an open Universe are instead limited by the last scattering surface. The physical meaning of this limit in the indetermination is that, from a classical point of view, there can be no sources placed ideally at infinity, making the plane-wave solution only but an artifact. One may think that only spherical modes, according to Huygen's principle, propagate in a finite space within a finite time. A different upper limit in the indetermination of an OAM state can be derived from superstring theory, characterized by a finite string length or from larger scales of space-time fuzziness expected from submillimeter gravity theories. Experimental results confirm anyway that the upper limit is closer to Planck's scale [46].

\section{CONCLUSIONS}

Our relativistic approach shows that Heisenberg's principle for OAM of light presents close analogies with Ehrenfest's paradox. We show that there are finite limits for the determination of OAM states of light dictated by the Hubble horizon of the Universe and by the finiteness of Planck units, below which space and time are not defined. The maximum value of spectral values one can achieve is on the order of $\ell \approx 10^{61}$ when the wavelength is on the order of the visible Universe Hubble horizon and the twisting step is measured in a time interval on the order of the Planck scale. The momentary rotation velocity remains subluminal within the Hubble horizon and the indetermination depends on the geometry of spacetime and on the choice of the reference frame of the observer because, being an exquisitely quantum phenomenon referred to the quantum properties of OAM photons, angles used to define the Heisenberg principle with OAM states have a purely formal value. Ideally, one might think to use OAM states either to determine the Hubble horizon $H$ or the existence of a scale for quantum gravity larger than the Plank scale, by determining the boundaries of the indetermination 
values of OAM states found, which represents a direct link from the smaller to the larger scales existing in our Universe.

\section{ACKNOWLEDGMENTS}

F.T. gratefully acknowledges ZKM and Peter Weibel for the financial support.
[1] C. Cohen-Tannoudji, J. Dupont-Roc, and G. Grynberg, Photons and Atoms (Wiley-VCH Verlag, Berlin, 2007).

[2] F. Tamburini and D. Vicino, Phys. Rev. A 78, 052116 (2008).

[3] R. A. Beth, Phys. Rev. 50, 115 (1936).

[4] V. Garcés-Chávez, D. McGloin, M. D. Summers, A. FernandexNieves, G. C. Spalding, G. Cristobal, and K. Dholakia, J. Opt. A: Pure Appl. Opt. 6, S235 (2004).

[5] M. Babiker, W. L. Power, and L. Allen, Phys. Rev. Lett. 73, 1239 (1994).

[6] M. F. Andersen, C. Ryu, P. Clade, V. Natarajan, A. Vaziri, K. Helmerson, and W. D. Phillips, Phys. Rev. Lett. 97, 170406 (2006).

[7] V. B. Berestetskii, E. M. Lifzhitz, and L. P. Pitaevskii, Quantum Electrodynamics, 2nd ed. (Pergamon Press, Oxford, UK, 1980), Chap. 1.

[8] I. Bialynicki-Birula and Z. Bialynicka-Birula, Phys. Rev. Lett. 108, 140401 (2012).

[9] G. F. Calvo, A. Picón, and E. Bagan, Phys. Rev. A 73, 013805 (2006).

[10] A. Mair, A. Vaziri, G. Weihs, and A. Zeilinger, Nature 412, 313 (2001).

[11] A. T. O’Neil, I. MacVicar, L. Allen, and M. J. Padgett, Phys. Rev. Lett. 88, 053601 (2002).

[12] J. Leach, M. J. Padgett, S. M. Barnett, S. Franke-Arnold, and J. Courtial, Phys. Rev. Lett. 88, 257901 (2002).

[13] J. Leach, J. Courtial, K. Skeldon, S. M. Barnett, S. FrankeArnold, and M. J. Padgett, Phys. Rev. Lett. 92, 013601 (2004).

[14] O. Emile, R. Niemiec, C. Brousseau, J. Emile, K. Mahdjoubi, W. Wei, and B. Thide, Eur. Phys. J. D 70, 172 (2016).

[15] F. Tamburini, E. Mari, B. Thidé, C. Barbieri, and F. Romanato, Appl. Phys. Lett. 99, 204102 (2011).

[16] F. Tamburini, E. Mari, A. Sponselli, B. Thidé, A. Bianchini, and F. Romanato, New J. Phys. 14, 033001 (2012).

[17] F. Tamburini, B. Thidé, and M. Della Valle, MNRAS Lett. 492, L22 (2019).

[18] B. Thidé, Electromagnetic Field Theory (Dover Publications, Mineola, NY, 2011).

[19] J. P. Torres and L. Torner, Twisted Photons: Applications of Light with Orbital Angular Momentum (Wiley-VCH, Weinheim, Germany, 2011).

[20] M. Beck, D. T. Smithey, J. Cooper, and M. G. Raymer, Opt. Lett. 18, 1259 (1993).

[21] J. A. Vaccaro and D. T. Pegg, J. Mod. Opt. 37, 17 (1990).
[22] S. Franke-Arnold, S. M. Barnett, E. Yao, J. Leach, J. Courtial, and M. Padgett, New J. Phys. 6, 103 (2004).

[23] G. Vallone, G. Parisi, F. Spinello, E. Mari, F. Tamburini, and P. Villoresi, Phys. Rev. A 94, 023802 (2016).

[24] C. H. J. Schmitz, K. Uhrig, J. P. Spatz, and J. E. Curtis, Opt. Express 14, 6604 (2006).

[25] B. Thidé, H. Then, J. Sjöholm, K. Palmer, J. E. S. Bergman, T. D. Carozzi, Y. N. Istomin, N. H. Ibragimov, and R. Khamitova, Phys. Rev. Lett. 99, 087701 (2007).

[26] L. Mandel and E. Wolf, Optical Coherence and Quantum Optics (Cambridge University Press, New York, 1995).

[27] H. A. Kastrup, Phys. Rev. A 73, 052104 (2006).

[28] L. D. Landau and E. M. Lifshitz, The Classical Theory of Fields, 4th ed. (Pergamon Press, Oxford, UK, 1995).

[29] A. Einstein, The Meaning of Relativity, Revised edition (Princeton University Press, NJ, 2014).

[30] P. Ehrenfest, Phys. Z. 10, 918 (1909).

[31] J. Stachel, in General Relativity and Gravitation: One Hundred Years after the Birth of Albert Einstein, edited by A. Held (Plenum Press, New York, London, 1980), Vol. 1, p. 1.

[32] H. Ishihara, M. Takahashi, and A. Tomimatsu, Phys. Rev. D 38, 472 (1988).

[33] F. Tamburini, B. Thidé, I. Licata, F. Bouchard, and E. Karimi, arXiv: 1707.07160

[34] J. Strohaber, Gen Relativ, Gravit. 45, 2457 (2013).

[35] J. Strohaber, arXiv:1807.00933.

[36] M. Tsamparlis, in Special Relativity (Springer-Verlag, Berlin, Heidelberg, 2010), pp. 495-519.

[37] H. Nikolić, Phys. Rev. A 61, 032109 (2000).

[38] R. P. Kerr, Phys. Rev. Lett. 11, 237 (1963).

[39] Edited by D. L. Wiltshire, M. Visser, and S. M. Scott, The Kerr Spacetime: Rotating Black Holes in General Relativity (Cambridge University Press, Cambridge, UK, 2009).

[40] F. Tamburini, B. Thide, G. Molina-Terriza, and G. Anzolin, Nat. Phys. 7, 195 (2011).

[41] S. Chandrasekhar and J. Wright, PNAS 47, 341 (1961).

[42] F. C. Speirits, M. P. J. Lavery, M. J. Padgett, and S. M. Barnett, Opt. Lett. 39, 2944 (2014).

[43] S. W. Hawking, Phys. Rev. D 46, 603 (1992).

[44] P. Pitanga, arXiv: 1110.0173.

[45] M. P. J. Lavery, S. M. Barnett, F. C. Speirits, and M. J. Padgett, Optica 1, 1 (2014).

[46] F. Tamburini, C. Cuofano, M. Della Valle, and R. Gilmozzi, Astron. Astrophys. 533, A71 (2011). 